\title{
Improved Closed-Loop Communication in the Presence of Feedback Delay and Error
}

\author{
Abdorreza Heidari and Amir K. Khandani \\ Coding and Signal Transmission Laboratory (www.cst.uwaterloo.ca) \\ The Department of Electrical and Computer Engineering \\ University of Waterloo, Waterloo, Ontario, Canada N2L 3G1 \\ Emails: \{reza, khandani\}@cst.uwaterloo.ca
}

\begin{abstract}
The closed-loop transmit diversity technique is used to improve the performance of the downlink channel in MIMO communication systems. The WCDMA standard endorsed by 3GPP adopts two modes of downlink closed-loop schemes based on partial channel state information. The information is fed back from the mobile unit to the base station through a low-rate uncoded feedback bit stream. Previously [1], we addressed the efficient reconstruction of the beamforming weight in the presence of feedback error, with the constraint of a constant transmit power.

In this article, the issue of feedback delay is also considered. Using joint source-channel coding techniques, a reconstruction algorithm is introduced to improve the performance of mode 1 of $3 \mathrm{GPP}$ in the presence of feedback error and delay, by taking advantage of the redundancy available in the bitstream of channel state information. We also introduce the novel concept of Blind Antenna Verification. It can substitute the conventional Antenna Weight Verification process without the need to any training data. The performance is examined within a simulated 3GPP framework. It is demonstrated that the proposed algorithms have substantial gain over the conventional method for low, moderate and high mobile speeds. The proposed approaches are applicable to other feedback schemes as well ${ }^{1}$.
\end{abstract}

\section{INTRODUCTION}

The increasing demand for internet and wireless services highlights the need for an increase of the capacity of the communication systems. Third generation of mobile communication, namely 3GPP [2] and 3GPP2 [3] have developed the WCDMA [4] and CDMA2000, respectively, to address this trend. The improvement of the downlink capacity is one of the main challenges of the $3 \mathrm{G}$ systems, and closed-loop techniques are known

\footnotetext{
${ }^{1}$ This work is financially supported by Bell Canada, Communications and Information Technology Ontario (CITO), and Natural Sciences and Engineering Research Council of Canada (NSERC).
}

to have the potential to solve the problem. Transmit Adaptive Array [5] is a part of the 3GPP standard with two transmit antennas at the base station and one receive antenna at the mobile unit, which uses the Channel State Information (CSI) to beamform the transmit signal.

The feedback data is a low-rate stream of quantized CSI, which is uncoded. Hence, the scheme is sensitive to the feedback error. Also like all closed-loop schemes, it is vulnerable to feedback delay, especially at high mobile speeds. Furthermore, the reconstruction scheme suggested by the standard is not efficient. Our focus here is on Mode 1 of 3GPP [6] which only feedbacks the phase information of the channel with a special quantization scheme. Mode 1 has a good performance at low mobile speeds, but it fails at higher speeds. In the previous work [1], the efficient reconstruction of beamforming weight in the presence of feedback error is addressed. Some joint source-channel techniques are used to improve the performance of Mode 1 of $3 \mathrm{GPP}$, by taking advantage of the redundancy available in the CSI stream.

In this article, the issue of feedback delay is also considered. We propose two approaches. One approach uses the previous method for the feedback error and uses predicted channel values to compensate for the effect of feedback delay. In the other approach, a unified joint source-channel coding platformframework deals with the feedback imperfection. Exploiting the novel concept of Blind Antenna Verification, this method only uses the feedback bitstream and does not need any preamble. The performance is examined in a simulated closedloop system compatible with the 3GPP standard. For the channel model, we consider independent Rayleigh fading channel and for simulating the mobile fading channel, we use a modified Jakes fading generator [7] to produce stationary fading signals. 


\section{A. Beamforming}

In a closed-loop system, channel input $\mathbf{x}$ should be appropriately selected according to the channel state [8]. Controlling the channel input can be accomplished with a conventional beamformer which applies some weights on the transmitted signal for each antenna. Assuming two transmit antennas and one receive antenna, a beamforming scheme can be expressed as

$$
\mathbf{x}=\mathbf{w} s,
$$

where

$$
\mathbf{w}=\left[w^{(1)}, w^{(2)}\right]^{T} \in \mathbb{C}^{2},
$$

and $s$ is the transmitted symbol. It is assumed that $\|\mathbf{w}\|^{2}=1$, which means the beamformer does not change transmit power. The received signal $r$ is a complex number which is the superposition of the signals from different channels, as well as the noise, i.e.,

$$
r=\mathbf{h}^{T} \mathbf{w} s+\eta=\left(\sum_{m=1}^{2} h^{(m)} w^{(m)}\right) s+\eta .
$$

In the decoding process, the combining variable $v \in \mathbb{C}$ is applied as [9]

$$
z=v^{H} r=v^{H} \mathbf{h}^{T} \mathbf{w} s+v^{H} \eta,
$$

where $z$ is used to calculate the output LLR (LogLikelihood Ratio) values. It has been shown [10] that to minimize the average probability of error in a MIMO system, w and $v$ should be jointly selected to maximize the instantaneous SNR. It could be characterized from (3) that $\mathrm{SNR}_{\text {inst }}=\left|\mathbf{h}^{T} \mathbf{w}\right|^{2} \frac{E_{s}}{N_{0}}$, where $E_{s}=$ $E\left[|s|^{2}\right]$. Later, we will talk about the selection of $v$ in the Antenna Verification section. Several schemes for selection of $\mathbf{w}$ are introduced in the literature, for example, refer to [8], [1].

\section{EFFICIENT RECONSTRUCTION OF THE BEAMFORMING WEIGHT}

In the sequel, we assume the framing structure and the quantization scheme of the closed-loop mode 1 of the 3GPP standard [6], [1]. However, our approach can be used for other feedback schemes as well.

It is assumed that $w_{n}^{(1)}=\frac{1}{\sqrt{2}}$ and $\left|w_{n}^{(2)}\right|=\frac{1}{\sqrt{2}}$, which come from the fact that the transmit power is constant and only phase information is fed back. Mode 1 suggests a linear combination to produce $\hat{w}_{n}^{(2)}$ at the base station. However, we suggest more efficient reconstruction algorithms.

Fig. 1 shows the quantization process in the receiver and the reconstruction process in the transmitter. $\tilde{\phi}_{n}$ is the quantized co-phase, and $I_{n}$ is the respective index where $I_{n} \in\{0,1,2,3\}$ corresponding to $\tilde{\phi}_{n} \in$ $\{-\pi / 2,0, \pi / 2, \pi\}$, respectively. $I_{n}$ is sent through the feedback channel and $J_{n}$ is received at the base station (noisy and delayed). Here, a memoryless feedback channel is assumed.

\section{A. MMSE Solution in the Presence of Feedback Delay and Error}

In the previous work [1], [11], the MMSE algorithm in the presence of erroneous (or noisy) feedback is introduced. The best algorithm is obtained when softoutput is used, and it is called SoftNMMSE. In the following, a similar approach is pursued to find an algorithm when feedback data is delayed as well.

Assuming a delay of $d$ symbols in the feedback channel ( $d$ is non-negative integer), the sequence $J_{n-d}=$ $\left[J_{1}, J_{2}, \cdots, J_{n-d}\right]$ is available at the base station at time $n$. The fundamental theorem of estimation states that given $\underline{J}_{n-d}$,

$$
\check{w}_{n}^{(2)}=E\left[w_{n}^{(2)} \mid \underline{J}_{n-d}\right]
$$

is the minimum mean-squared error (MMSE) estimate of the weight $w_{n}^{(2)}$. Similar to what is shown in [12], the formula can be approximated by the following feasible form:

$$
\check{w}_{n}^{(2)}=\sum E\left[w_{n}^{(2)} \mid \underline{I}_{n}^{n-\mu+1}\right] P\left(\underline{I}_{n}^{n-\mu+1} \mid \underline{J}_{n-d}\right),
$$

where the summation is over all the possible $\mu$-fold sequences of $\underline{I}_{n}^{n-\mu+1}=\left[I_{n-\mu+1}, \cdots, I_{n-1}, I_{n}\right]$. In the MMSE sense, the formula is asymptotically optimum for sufficiently large values of $\mu$. In (6), $E\left[w_{n}^{(2)} \mid \underline{I}_{n}^{n-\mu+1}\right]$ is a codebook, and the probability part can be computed as explained in Section II-A.1.

We are dealing with the estimation of a complex variable with a constant amplitude, as $\left|\hat{w}_{n}^{(2)}\right|=\frac{1}{\sqrt{2}}$. However, there is no control on the amplitude of $\check{w}_{n}^{(2)}$ in (5) and (6). Hence, we need an MMSE estimator with a constant amplitude, which is introduced in a lemma in [1]. According to the lemma, the antenna weight can be calculated using the MMSE solution of (5) or (6) as

$$
\hat{w}_{n}^{(2)}=\frac{1}{\sqrt{2}} \frac{\check{w}_{n}^{(2)}}{\left|\check{w}_{n}^{(2)}\right|} .
$$

1) Markov Model: For capturing the residual redundancies [13] in the feedback bitstream, we assume that the bitstream follows a Markov model of order $\gamma$. A trellis structure is set up based on the Markov model 


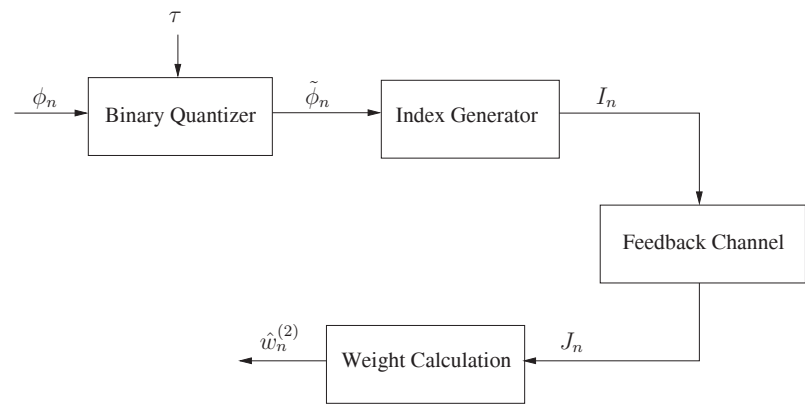

Fig. 1. Block diagram of encoding and decoding of the quantizer system

to exploit the redundancies. The states of the trellis are defined as $S_{n}=\underline{I}_{n}^{n-\gamma+1}$. The trellis is specified by the probabilities of the state transitions, $P\left(S_{n} \mid S_{n-1}\right)$, which are the A Priori Probabilities (APP) of the Markov model.

The probability $P\left(\underline{I}_{n}^{n-\mu+1} \mid \underline{J}_{n-d}\right)$ could be calculated by using the state probabilities given the received feedback data, $P\left(S_{n} \mid \underline{J}_{n-d}\right)$. Appendix I provides a recursive algorithm for calculation of $P\left(S_{n} \mid \underline{J}_{n-d}\right)$. Note that this is essentially a kind of prediction as the last $d$ feedback symbols are not available due to the feedback delay. The resulting algorithm is called SoftNMMSE-JP here.

\section{B. Channel Prediction at the Receiver}

Another approach to overcome the feedback delay is introduced here. If the receiver calculates the feedback symbols by using the future channel states, it can proactively cancel out the effect of the delay in the feedback channel. This approach can be implemented by using $d$ step predicted values of the channel coefficients. These values can be achieved by applying a short-range fading prediction algorithm [14], [15] to the current channel values. Here we use an Auto Regressive (AR) model of order 3 to predict the fading samples, and SoftNMMSE is used to deal with the feedback error. The resulting algorithm is called SoftNMMSE,LP.

In practice, channel coefficients are estimated using some pilots, training bits, etc. which usually introduces some error in the available channel coefficients. Note that these channel estimates are used to generate feedback symbols, calculate the LLR values for the decoding process, etc. Therefore, the quality could have a direct impact on the overall performance of a closed-loop system. Here, the channel estimation error is modelled as an Additive White Gaussian Noise (AWGN), and $\mathrm{SNR}_{z}$ is defined as the ratio of the channel power to the noise power. The effect of $\mathrm{SNR}_{z}$ on the performance of different algorithms is examined in the simulations.

\section{Antenna Weight VerificAtion}

When an error occurs in the feedback channel, an incorrect antenna weight (i.e., beamforming weight) vector is applied at the transmitter, which leads to two consequences. First, the received signal power is smaller [16], because a non-optimum weight is applied. However, our simulations show that the performance degradation due to this effect is rather small. The second consequence is much more serious: Each time a feedback error occurs, the mobile station does not know the actual antenna weight vector that is applied at the base station. Since the mobile station obtains the dedicated (i.e., userspecific) channel estimate by combining the estimates for individual antennas from common pilots with the assumed weight vector used at the base station. This causes serious dedicated channel estimation error, resulting in some error floor in the performance. To minimize the effect of the problem, a technique called Antenna Weight Verification (AV) [17] has been suggested. In this method, some extra training bits are required. In other words, certain dedicated preamble bits should be transmitted to all users.

In the next section, we introduce a novel technique called Blind Antenna Verification (BAV) which does not need any extra preamble bits. Using BAV in conjunction with the SoftNMMSE-JP algorithm provides a powerful closed-loop algorithm in the presence of feedback imperfections.

\section{A. Blind Antenna Verification}

First of all, note that

$$
\mathbf{w}_{n} \leftrightarrow \underline{I}_{n-d} \leftrightarrow \underline{J}_{n-d}
$$

constitute a Markov chain, as the information flow shows.

At the transmitter, the best estimate of the optimum beamforming weight, in the MMSE sense, is

$$
\hat{\mathbf{w}}_{n}=E\left[\mathbf{w}_{n} \mid \underline{J}_{n-d}\right] .
$$

Transmitter applies the weight, and the receiver should find the best estimate of the $\hat{\mathbf{w}}_{n}$ to calculate the dedicated channel estimate to do the decoding process (which is the BAV goal). Assume that the receiver uses the following estimate,

$$
\tilde{\mathbf{w}}_{n}=E\left[\mathbf{w}_{n} \mid \underline{I}_{n-d}\right] .
$$

Therefore, considering the BAV goal, the transmitter should apply a weight as close to $\tilde{\mathbf{w}}_{n}$ as possible. The 
transmitter can estimate the $\tilde{\mathbf{w}}_{n}$ given its feedback data $\underline{J}_{n-d}$. Then, the MMSE estimate is as follows

$$
\begin{aligned}
& E\left[\tilde{\mathbf{w}}_{n} \mid \underline{J}_{n-d}\right]= \\
& \quad=E\left[E\left[\mathbf{w}_{n} \mid \underline{I}_{n-d}\right] \mid \underline{J}_{n-d}\right] \\
& \quad=E\left[\mathbf{w}_{n} \mid \underline{J}_{n-d}\right] \\
& \quad=\hat{\mathbf{w}}_{n}
\end{aligned}
$$

where Lemma 1 is used to reach (13). The result show that this estimate is the same as the optimum weight at the transmitter. In other words, if the transmitter uses $\hat{\mathbf{w}}_{n}$, and the receiver uses $\tilde{\mathbf{w}}_{n}$, both the optimum beamforming and BAV goals are achieved.

For implementation of $\mathrm{BAV}$, the receiver calculates (10) which could be done similar to (6). However, it does not need any trellis processing because the probabilities are fixed. The required $P\left(S_{n} \mid \underline{I}_{n-d}\right)$ can be calculated as shown in Appendix II.

\section{NumERICAL RESUltS}

The block diagram of the simulated feedback system is shown in Fig. 2. The simulation parameters are the same as [1]. A feedback delay of $d=2$, and a feedback error of 5\% (or the equivalent Additive White Gaussian Noise for the soft-output, $\sigma_{n}=0.6080$ ) is assumed. The following algorithms are compared: Standard (mode 1 of 3GPP), "SoftNMMSE-JP, BAV", "SoftNMMSE, LP", and "SoftNMMSE, IP". The last algorithm uses the same approach as Section II-B but assumes Ideal channel Prediction, i.e., it assumes that the required future channel coefficients are perfectly available, which is considered as a performance bound.

Fig. 3 and Fig. 4 show the FER performance versus transmit SNR at four different mobile speeds for $\mathrm{SNR}_{z}=40 \mathrm{~dB}$ and $\mathrm{SNR}_{z}=10 \mathrm{~dB}$, respectively. It is observed that both approaches significantly outperform the Standard algorithm at all mobile speeds. For the case of high $\mathrm{SNR}_{z}$ (for example, when powerful channel pilots are available), the channel prediction approach acts better than the other approach which only uses the feedback bitstream. However, when $\mathrm{SNR}_{z}$ is low, it is the opposite, since the predicted channel values are not very accurate in this case.

\section{ApPEndices}

A. Appendix I: Derivation of $P\left(S_{n} \mid \underline{J}_{n-d}\right)$ for Transmitter

$$
P\left(S_{n} \mid \underline{J}_{n-d}\right)=C_{1} \cdot P\left(S_{n}, \underline{J}_{n-d}\right)
$$

$$
\begin{aligned}
= & C_{1} \cdot P\left(\underline{J}_{n-d-1}\right) \cdot P\left(S_{n} \mid \underline{J}_{n-d-1}\right) \\
& \cdot P\left(J_{n-d} \mid S_{n}, \underline{J}_{n-d-1}\right) \\
= & C \cdot P\left(S_{n} \mid \underline{J}_{n-d-1}\right) \cdot P\left(J_{n-d} \mid I_{n-d}\right)
\end{aligned}
$$

for $d \leq \gamma-1$, where $C$ and $C_{1}$ are normalizing variables. $P\left(S_{n} \mid \underline{J}_{n-d-1}\right)$ can be written as follows

$$
\begin{aligned}
P\left(S_{n} \mid \underline{J}_{n-d-1}\right)= \\
\quad \underline{\underline{S}}_{n-1} \cdots \sum P\left(S_{n} \mid \underline{S}_{n-1}, \underline{J}_{n-d-1}\right) \cdot P\left(\underline{S}_{n-1} \mid \underline{J}_{n-d-1}\right) \\
=\sum_{\underline{S}_{n-1}} \cdots \sum P\left(S_{n} \mid S_{n-1}\right) \cdot P\left(S_{n-1}, \underline{S}_{n-2} \mid \underline{J}_{n-d-1}\right) \\
=\sum_{S_{n-1}} P\left(S_{n} \mid S_{n-1}\right) \cdot \sum \cdots \sum P\left(S_{n-1}, \underline{S}_{n-2} \mid \underline{S}_{n-d-1}\right) \\
=\sum_{S_{n-1}} P\left(S_{n} \mid S_{n-1}\right) \cdot P\left(S_{n-1} \mid \underline{J}_{n-d-1}\right)
\end{aligned}
$$

Therefore, $P\left(S_{n} \mid \underline{J}_{n-d}\right)$ can be calculated recursively as

$$
\begin{aligned}
& P\left(S_{n} \mid \underline{J}_{n-d}\right)=C \cdot P\left(J_{n-d} \mid I_{n-d}\right) \\
& \quad \cdot \sum_{S_{n-1}} P\left(S_{n} \mid S_{n-1}\right) \cdot P\left(S_{n-1} \mid \underline{J}_{n-d-1}\right)
\end{aligned}
$$

B. Appendix II: Derivation of $P\left(S_{n} \mid \underline{I}_{n-d}\right)$ for Receiver

$$
P\left(S_{n} \mid \underline{I}_{n-d}\right)=P\left(S_{n} \mid \underline{S}_{n-d}\right)=P\left(S_{n} \mid S_{n-d}\right),
$$

where $P\left(S_{n} \mid S_{n-d}\right)$ is required to be calculated once, as follows

$$
\begin{aligned}
& P\left(S_{n} \mid S_{n-d}\right)=P\left(S_{n} \mid \underline{S}_{n-d}\right) \\
& \left.=\sum_{\underline{S}_{n-1}^{n-d-1}} \cdots \sum_{n} \mid S_{n-1}\right) . \\
& \quad P\left(S_{n-1} \mid S_{n-2}\right) \cdots P\left(S_{n-d-1} \mid S_{n-d}\right)
\end{aligned}
$$

\section{Appendix III: Lemma 1}

Lemma 1: Assume that $X \leftrightarrow Y \leftrightarrow Z$ constitute a Markov chain. Then,

$$
E[E[X \mid Y] \mid Z]=E[X \mid Z] .
$$

\section{REFERENCES}

[1] A. Heidari, F. Lahouti, and A. K. Khandani, "Improved Reconstruction of Channel State Information for Low-Rate Feedback Schemes," submitted to IEEE Trans. on Vehicular Technology, 2005.

[2] "The 3GPP website: http: / / www . 3gpp.org."

[3] "The 3GPP2 website: http: / /www . 3gpp2 .org." 


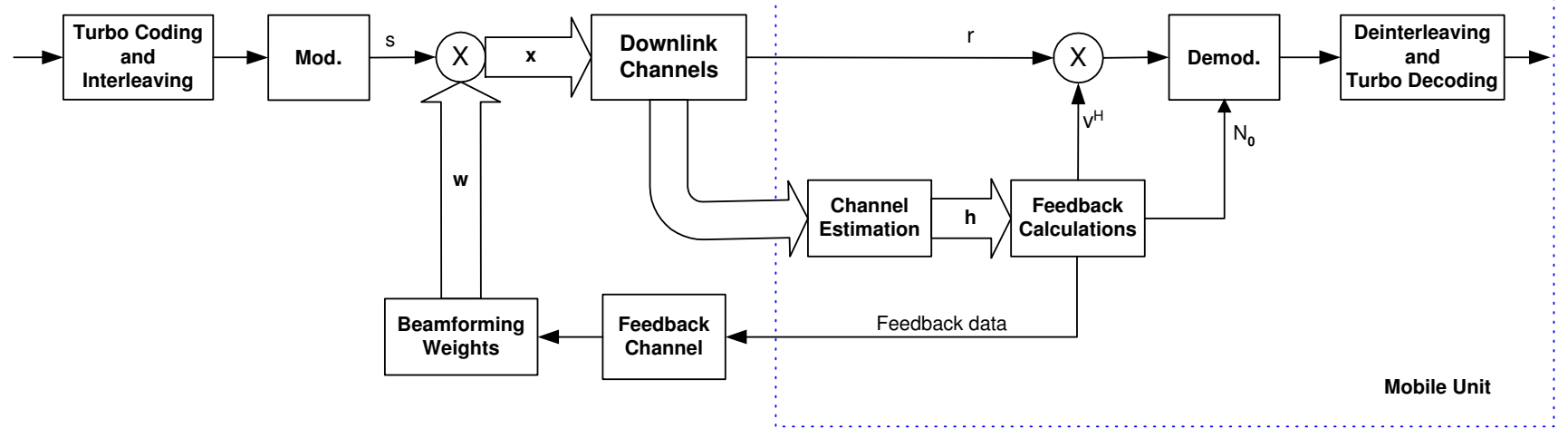

Fig. 2. Block diagram of our feedback system
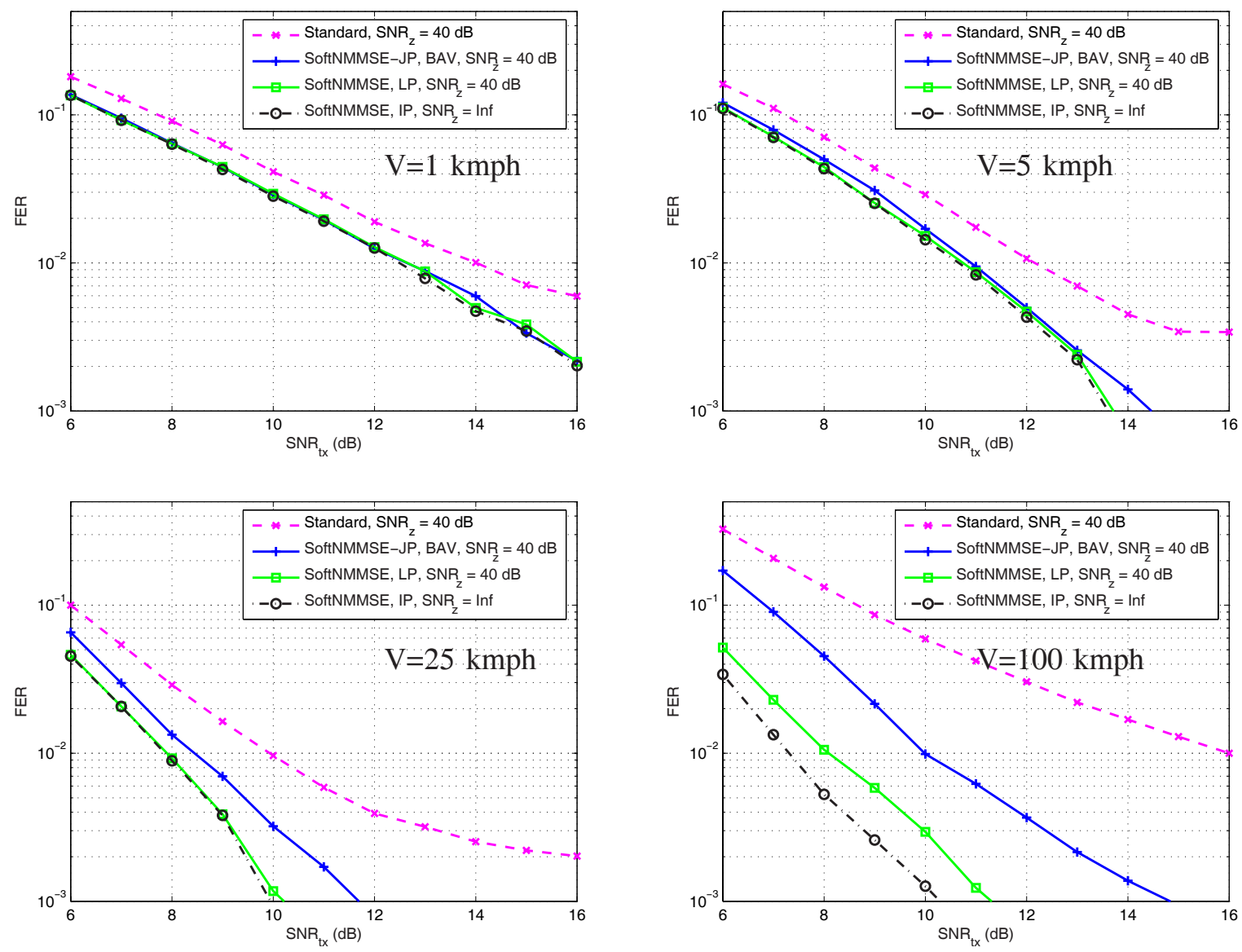

Fig. 3. FER performance at $\mathrm{SNR}_{z}=40 \mathrm{~dB}$ 

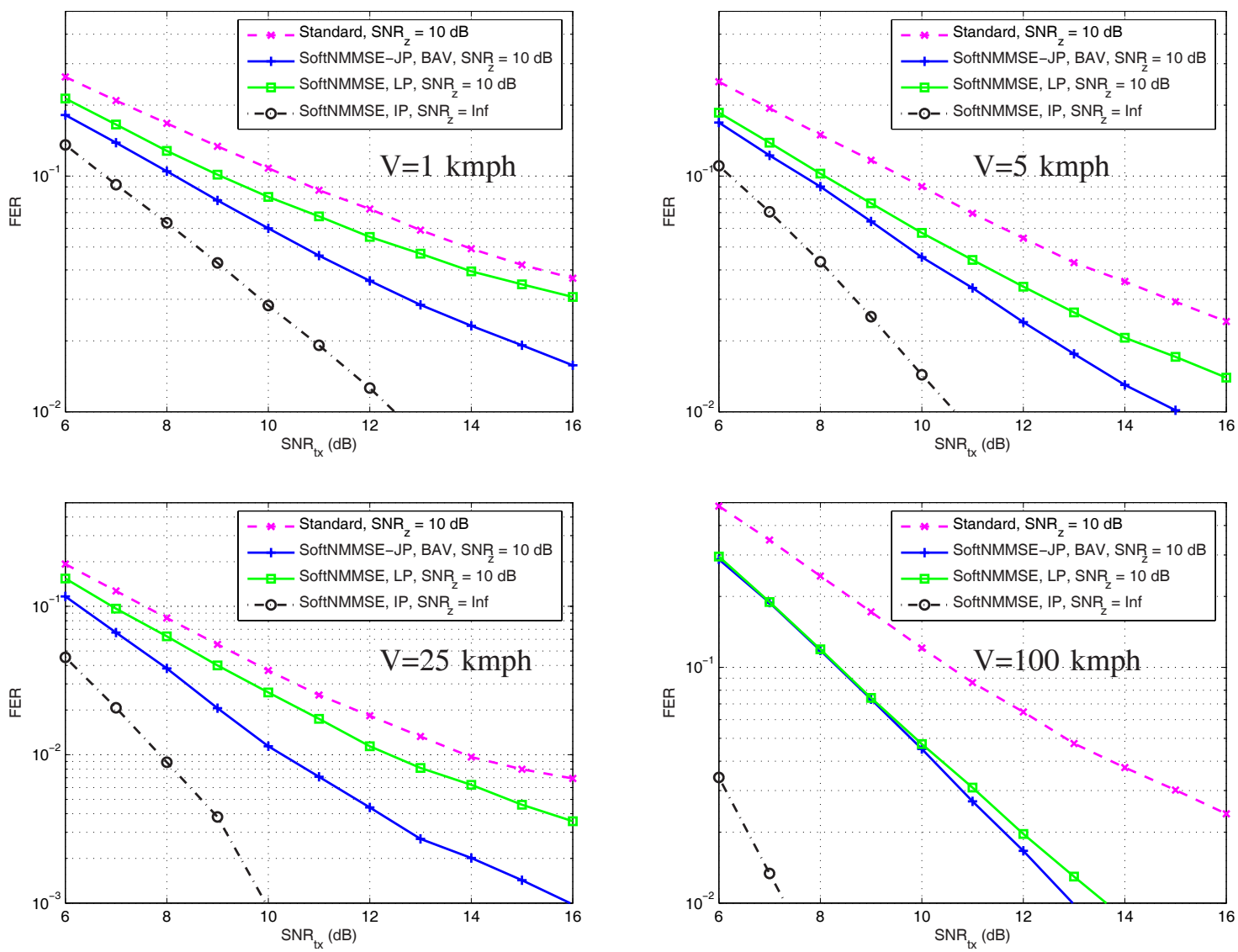

Fig. 4. FER performance at $\mathrm{SNR}_{z}=10 \mathrm{~dB}$

[4] 3GPP Technical Specification, "UMTS Physical Layer: General Description," ETSI TS 125201 V6.0.0, Dec. 2003.

[5] R. T. Derryberry et al., "Transmit Diversity in 3G CDMA Systems," IEEE Communications Magazine, pp. 68-75, Apr. 2002.

[6] 3GPP Technical Specification, "UMTS Physical Layer Procedures (FDD)," ETSI TS 125214 V6.0.0, Dec. 2003.

[7] M. F. Pop and C. Beauliu, "Limitations of Sum-of-Sinusoids Fading Channel Simulators," IEEE Trans. on Communications, pp. 699-708, Apr. 2001.

[8] A. Narula, M. J. Lopez, M. D. Trott, and G. W. Wornell, "Efficient Use of Side Information In Multiple-Antenna Data Transmission Over Fading Channels," IEEE Journal on selected Areas in Communications, pp. 1423-1436, Oct. 1998.

[9] J. G. Proakis, Digital Communications. Mc-Graw Hill International Editions, 2001.

[10] P. A. Dighe, R. K. Mallik, and S. S. Jamuar, "Analysis of Transmit-Receive Diversity in Rayleigh Fading," IEEE Trans. on Communications, pp. 694-703, Apr. 2003.

[11] A. Heidari, F. Lahouti, and A. K. Khandani, "Improved Reconstruction of Channel State Information in 3GPP," Vehicular Technology Conference (VTC'F05), Dallas, Texas, USA, Sept. 2005.

[12] F. Lahouti and A. K. Khandani, "Reconstruction of Predic- tively Encoded Signals over Noisy Channels Using a Sequence MMSE Decoder," IEEE Trans. on Communications, pp. 12921301, Aug. 2004

[13] K. Sayood and J. C. Brokenhagen, "Use Of Residual Redundancy In The Design Of Joint Source-Channel Coders," IEEE Trans. on Communications, pp. 838-846, 1991.

[14] A. Duel-Hallen, S. Hu, H. Hallen, "Long Range Prediction of Fading Signals: Enabling Adaptive Transmission for Mobile Radio Channels," IEEE Signal Processing Magazine, May 2000.

[15] A. Heidari, F. Lahouti, D. McAvoy, and A. K. Khandani, "Improvement of Closed-loop Communication Systems in the Presence of Feedback Imperfections," tech. rep., Bell Mobility, Dec. 2005.

[16] H. Gerlach, "SNR Loss due to Feedback Quantization and Errors in Closed Loop Transmit Diversity Systems," The 13th IEEE International Symposium on Personal, Indoor and Mobile Radio Communications (PIMRC), pp. 2117-2120, Sept. 2002.

[17] A. Seeger and M. Sikora, "Antenna Weight Verification for Closed-Loop Transmit Diversity," IEEE Global Telecommunications Conference (GLOBECOM), pp. 1124-1129, Dec. 2003. 